\title{
Interference Mitigation in MC-CDMA System with CFO compensation based Modified MMSE-FDE Technique
}

\author{
Akshay Agnihotri \\ Vellore Institute of Technology \\ VIT University \\ India
}

\begin{abstract}
Multi-carrier Code Division Multiple Access (MC-CDMA) has been an attractive technique for wireless communication because it offers high data rate, high spectral efficiency, large system capacity, less transmit power and less implementation complexity. But due to Carrier Frequency Offset (CFO), which comes into account due to asynchrony between transmitter and receiver local oscillators or by Doppler Effect due to high mobility, orthogonality among the subcarriers gets effected resulting in Inter Carrier Interference (ICI) which leads to rapid degradation in performance of MC-CDMA system. In this paper, the performance of Uplink MC-CDMA system with Zero forcing- frequency domain Equalization (ZF-FDE), conventional MMSE equalizer and Modified MMSE-FDE using Walsh-Hadamard spreading sequence in multipath fading channel has been analyzed. Simulation has been performed using 32 subcarriers and 64 subcarriers for these equalization techniques. Simulation results confirm excellent performance of the Modified MMSE-FDE which outperforms the conventional MMSE equalization and ZFFDE. The further improvement in performance is achieved by employing 64 subcarriers instead of 32 subcarriers. Numerical equations and simulation results are given to support the analysis.
\end{abstract}

\section{Keywords}

MC-CDMA, CFO, MMSE-FDE, ZF, ICI, Walsh-Hadamard Sequence.

\section{INTRODUCTION}

MC-CDMA has always seen as an attention seeker for the next generation wireless communication due to its capability to provide high transmission rate as well as combating various channel effects like frequency-selective fading. Besides this, MC-CDMA also provides a high spectral efficiency, high flexibility in terms of data rate and most beneficiaries in its easy implementation with the help of FFT (Fast Fourier Transform) device without increasing the complexity of transmitter and receiver [1].

MC-CDMA adapts the beneficiary properties of both Code Division Multiple Access (CDMA) as well as Orthogonal Division Multiple Access (OFDM). As OFDM employs large number of orthogonal subcarriers to transmit user data in parallel, symbol duration get increased which helps in combating multipath interference [1]. Though OFDM provides a high data transmission rate, it suffers from lack of frequency diversity. To overcome this problem MC-CDMA is employed, which spreads user data along subcarriers. But like OFDM, MC-CDMA is sensitive to CFO, especially when number of carriers is large. Frequency offset is a small shift in broadcast carrier frequency, which mainly exists due to Doppler effects and/or due to the misalignment between transmitter and receiver oscillator [3]. Due to CFO orthogonality among the subcarriers is destroyed and thus Inter carrier Interference (ICI) and Multiuser Interference (MUI) are generated within the system.

Several methods have been proposed already to mitigate and compensate the effect of CFO. In [2] Younsun Kim, Sooyong Choi, Chulwoo You, and Daesik Hong and in [3] Jiho Jang and Kwang Bok (Ed) Lee have analyzed the effect of CFO on MC-CDMA using Equal gain combining (EGC) and Maximal ratio combining (MRC) equalizers. In [4] Youssef Nasser, Mathieu des Noes, Laurent Ros and Genevieve Jourdain have analyzed the sensitivity of multicarrier systems to synchronization errors. Similarly the effect of CFO on MCCDMA system has been discussed in [5] by Ying Li, Xiang Guiandin[6] by Luciano Tomba, Witold A. Krzymien, in which the performance of system with carrier phase noise and frequency offset has been analyzed. In [7] PH Reddy and VU Reddy have analyzed performance degradation of MC-CDMA at high Signal to noise ratio (SNR) using MMSE-equalization due to the Residual frequency offset (RFO).

In this paper a Modified Minimum Mean Square Error Frequency Domain Equalization (MMSE-FDE) technique is used to solve the ICI problem of MC-CDMA. To compensate the effect of CFO. Modified MMSE-FDE is compared with the conventional MMSE Equalization and ZF-FDE technique in terms of the Bit error rate (BER) performance for corresponding SNR's. Simulation results shows that Modified MMSE-FDE outperforms the conventional MMSE equalization as well as ZF-FDE techniques.

The paper is structured as follows. The MC-CDMA transmission model is described in Section II. In the next section, Section III, the Modified MMSE-FDE equalization scheme is presented. Followed by the performance simulation and comparison between the two schemes for different spreading codes in section IV and conclusion in Section V.

\section{SYSTEM MODEL}

Consider a MC-CDMA system with ' $U$ ' uplink users communicating with the Base Station (BS) through multipath channels. Consider total numbers of Subcarriers to be ' $M$ '. Walsh-Hadamard codes are employed in MC-CDMA for spreading the user data. The Walsh-Hadamard Matrix $\mathrm{W}^{\mathrm{M}}$ of size $\mathrm{M} * \mathrm{M}$ is given as,

$W^{M}=\left[\begin{array}{llll}\mathrm{w}_{0}^{M} & \mathrm{w}_{1}^{M} & \ldots & \mathrm{w}_{M-1}^{M}\end{array}\right]$ 
Where $w_{k}^{M}=\left[w_{0, k}^{M} w_{1, k \ldots}^{M} w_{M-1, k}^{M}\right]^{T}$ denotes transpose of a sequence vector. The transmitter block is shown in Figure 1. User data is modulated using Binary phase shift keying (BPSK) modulation. Let the data symbol after modulation is denoted by ' $\mathrm{x}$ '. After that the mapped data is passed through Serial to parallel converter (S/P). Then the parallel data is passed through the Spreading block, which spreads the data using Walsh- Hadamard sequence.

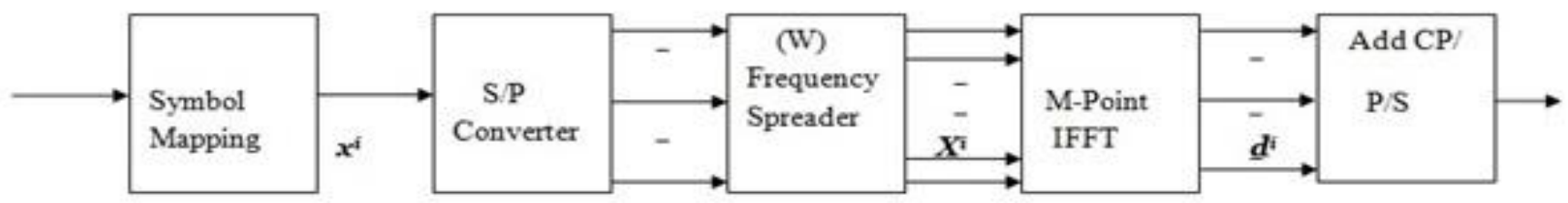

Fig 1: Transmitter of an MC-CDMA Uplink System

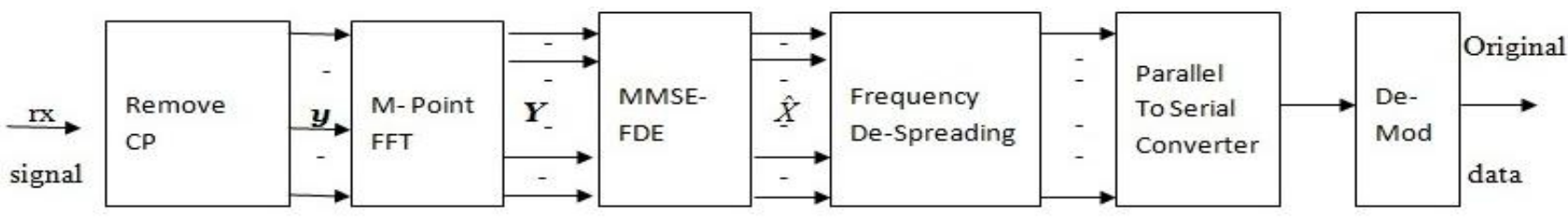

Fig 2: Receiver of an MC-CDMA Uplink System

The output of frequency spreader block is for $i^{\text {th }}$ user is given as,

$$
X^{i}=\sum_{k=0}^{M-1} W_{k}^{M} x^{k}
$$

Output of Frequency Spreader block is then passed to IFFT block, the resultant of which is given as,

$d^{i}=F_{I}^{i} X^{i}$

Where $F_{I}$ is an IFFT matrix, generated using equation,

$\left[F_{I}\right]_{p, q}=-\frac{1}{M} \exp \left(\frac{j 2 \pi p q}{M}\right)$

for $\mathrm{p}$ and $\mathrm{q}$ ranges from 0 to $\mathrm{M}-1$. Now a sufficiently long Cyclic-prefix (CP) of length (L-1) is appended at the beginning of each block, to avoid the Inter Block Interference (IBI). Now parallel-to-serial (P/S) conversion is performed and resulting signal is passed over the fading channel.

The Receiver block is shown in Figure.2. The received signal after passing through channel is considered as ' $r$ '. After removing $\mathrm{CP}$ the $\mathrm{i}^{\text {th }}$ user expression is given as,

$r^{i}=C^{i} d^{i}$

where $\mathrm{C}^{\mathrm{i}}$ is circulant channel matrix whose column vector is $\mathrm{c}^{i}=\left[\mathrm{c}_{0}^{i}, \mathrm{c}_{1}^{i}, \ldots \mathrm{c}_{\delta-1}^{i}, 0, \ldots . .0\right]^{T} \quad$ and $\delta$ represents maximum channel delay spread. It is also assumed that the different user equipment's have statistically independent channels. Now considering the effect of CFO and additive noise, then the received signal vector is given by,

$$
\begin{aligned}
& y=\sum_{u=1}^{U} O^{\zeta_{u}} r^{u}+z \\
& =\sum_{u=1}^{U} O^{\zeta_{u}} C^{u} d^{u}+z
\end{aligned}
$$

where $O^{\zeta_{u}}$ is $M \times M$ diagonal CFO matrix given as :

$O^{\zeta_{u}}=\operatorname{diag}\left\{1, \exp \left(\frac{j 2 \pi \zeta_{u}}{M}\right) \ldots \ldots, \exp \left(\frac{j 2 \pi(\mathrm{M}-1) \zeta_{u}}{M}\right)\right\}$

and $\zeta_{u}$ is the $\mathbf{u}^{\text {th }}$ user-equipment's normalized CFO. If $\zeta_{u}$ $=0$, that means there is no frequency offset i.e, receiver and transmitter oscillators are properly synchronized, then $O^{\zeta}=$ $\mathrm{I}_{M}$. ' $\mathbf{z}$ ' is white Gaussian noise vector. After this the signal is passed through M-point FFT, the output of which is given as

$$
\begin{aligned}
& Y=F_{F} y \\
& =F_{F}\left(\sum_{u=1}^{U} O^{\zeta_{u}} C^{u} d^{u}+z\right) \\
& =\sum_{u=1}^{U} F_{F} O^{\zeta_{u}} F_{F}{ }^{H} F_{F} C^{u} F_{F}{ }^{H} F_{F} d^{u}+z \\
& =\sum_{u=1}^{U} \Delta^{\zeta_{u}} \Omega^{u} D^{u}+Z
\end{aligned}
$$

where $\Delta^{\zeta_{u}}=F_{F} O^{\zeta_{u}} F_{F}{ }^{H}$ is the Circulant CFO matrix of User-equipment 'u' in frequency domain and $\Omega^{u}=$ $F_{F} C^{u} F_{F}{ }^{H}$ channel response in frequency domain and noise in frequency domain is $Z=F_{F} z$.

\section{MODIFIED "MMSE- FDE"TECHNIQUE}

In the case of conventional MMSE equalization and ZF-FDE, the residual MUI exist even after the CFO compensation, which eventually degrades the performance of system. In this paper Modified MMSE -FDE equalization technique is 
employed which considers the remaining MUI into account for designing the FDE coefficient.

The MMSE FDE is performed after the separating the individual user-equipment in order to suppress the remaining MUI for each user-equipment. Also the direct compensation is performed to eliminate the probabilities of self-interference caused due to the frequency offsets.

Now the detection of user-equipment1 and extraction of desired signal is performed, the signal vector after direct compensation for User-equipmentlis given as [9]:

$$
\begin{aligned}
& Y^{1}=F_{\mathrm{F}} O^{-\zeta_{u}} y \\
& =\Omega^{1} X^{1}+\sum_{u=2}^{U} \Delta^{\left(\zeta_{u}-\zeta_{1}\right)} \Omega^{u} X^{u}+F_{F} O^{-\zeta_{1}} Z
\end{aligned}
$$

$$
\text { (2) }
$$

Please where $O^{-\zeta_{u}}$ represents the CFO compensation of user-equipment1.In (14), the (i) represents the channel responded signal of user-equipment1 and (ii) represents MUI term which must be suppressed and the (iii) is additive noise vector. It is also noticed that the off-diagonal elements of the $\Omega^{1}$ are relatively smaller with respect to the diagonal ones, thus we can approximate it as a banded matrix [8].

By rearranging and redefining the parameters in (14), $\tilde{\Omega}^{u}=\Delta^{\left(\zeta_{u}-\zeta_{1}\right)} \Omega^{u} \quad$ and $Z^{1}=F_{F} O^{-\zeta_{1}} Z$ equation can rewritten as,

$$
Y^{1}=\tilde{\Omega}^{1} X^{1}+\sum_{u=2}^{U} \tilde{\Omega}^{u} X^{u}+Z^{1}
$$

Now equalization is performed on each user-equipment signal to mitigate the ICI and MUI. The FDE coefficient vector for conventional MMSE equalization technique is given as,

$$
\Psi_{\text {Conv. MMSE }}^{1}=\left(\tilde{\Omega}^{1}\right)^{H}\left[\tilde{\Omega}^{1}\left(\tilde{\Omega}^{1}\right)^{H}+\mathrm{V}_{Z^{1}}\right]^{-1}
$$

Where ()$^{\mathrm{H}}$ indicates the Hermitian operation and $\mathrm{V}_{Z^{1}}$ denotes the co-variance matrix of noise vector $Z^{1}$.Similarly the FDE coefficient vector for Zero Forcing equalization is given as,

$$
\Psi_{Z F}^{1}=\left(\tilde{\Omega}^{1}\right)^{H}\left[\tilde{\Omega}^{1}\left(\tilde{\Omega}^{1}\right)^{H}\right]^{-1}
$$

The Modified MMSE-FDE technique take into account the MUI term effectively in designing the FDE coefficient for each user-equipment. The conventional MMSE equalization technique doesn't taken into account the remaining MUI term. This is the main cause behind the enhanced performance of Modified MMSE-FDE in comparison to conventional MMSE.

The FDE coefficient for Modified MMSE-FDE is given as,

$$
\Psi^{1}=\left(\tilde{\Omega}^{1}\right)^{H}\left[\sum_{u=1}^{U} \tilde{\Omega}^{u}\left(\tilde{\Omega}^{u}\right)^{H}+N_{0} I_{M}\right]^{-1}
$$

where $N_{0}$ denoted the noise variance. The estimated data after equalization for Modified MMSE-FDE is given as,

$$
\hat{X}^{1}=\Psi^{1} Y^{1}
$$

After the estimation the signal vector is passed through the frequency de-spreader block, the resulting signal of which is then passed through the parallel to serial converter and Demodulator to get back the original data.

\section{SIMULATION RESULTS}

In Simulation part, the performance of ZF-FDE, conventional MMSE equalization and Modified MMSE-FDE has been analyzed using the Walsh-Hadamard spreading sequence. The channel used is the 4 path equal gain Rayleigh fading model. For an MC-CDMA uplink model M=32 and 64 subcarriers are considered for 4 user-equipment's. The normalized CFO's are assumed to be $\left[\zeta_{1}, \zeta_{2}, \zeta_{3}, \zeta_{4}\right]=[0.2,-0.05,0.3,-0.15]$ . Assumption made that the channel state information is known to BS.

Figure. 3 shows the BER performance of the ZF-FDE and Conventional MMSE equalization for ICI cancellation using Walsh- Hadamard spreading sequence using 32 sub-carriers. From Figure.3, it is shown that the performance of conventional MMSE equalization is better than the Zero forcing equalization, due to the reason that the zero forcing equalizer amplifies the noise along with the signal, which results in degrading its performance. So the conventional MMSE equalization perform better and achieves lower BER than the ZF-FDE.

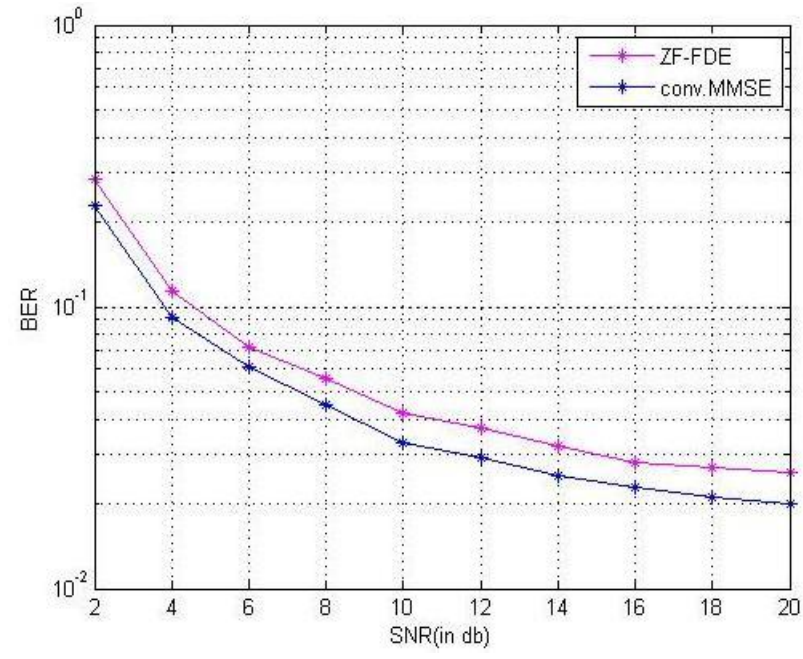

Fig 3: BER Performance of the ZF-FDE and conventional MMSE equalization using Walsh-Hadamard Sequence

Figure.4 and Figure.5 displays the BER performance of conventional MMSE equalization and Modified MMSE-FDE technique for 32-subcarriers and 64- subcarriers respectively and it is found that the Modified MMSE-FDE outperforms conventional MMSE equalization and achieves lower BER, which is mainly due to consideration of the remaining MUI term in Modified MMSE-FDE technique. From the Figure.5, it is noticeable that the performance of Modified MMSE-FDE has been improved by employing 64 subcarrier instead of 32 subcarrier in the system. This indicates that the MUI term is assumed to be reconstructed and cancelled out from the received signal. 


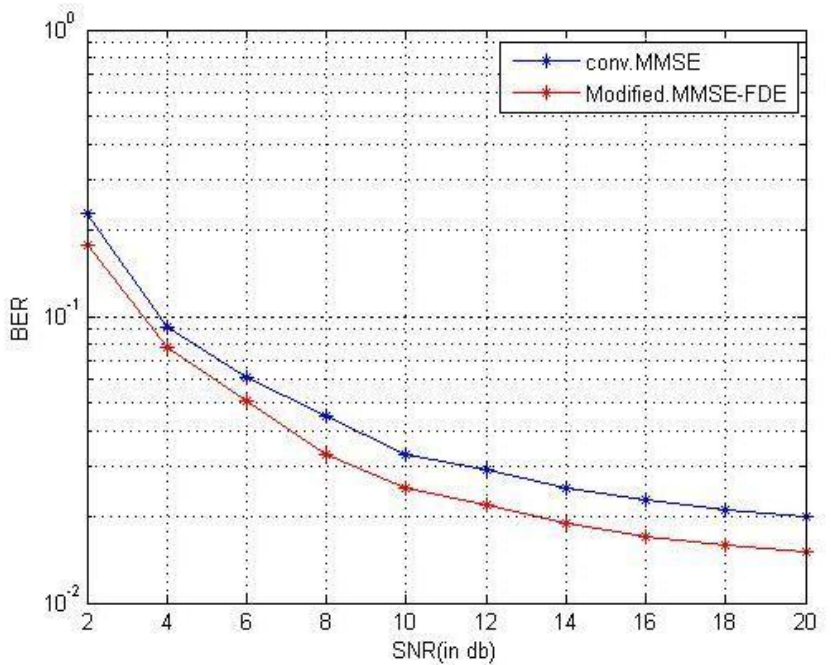

Fig 4: BER Performance of the conventional MMSE equalizer \& Modified MMSE-FDE technique for 32 Subcarriers.

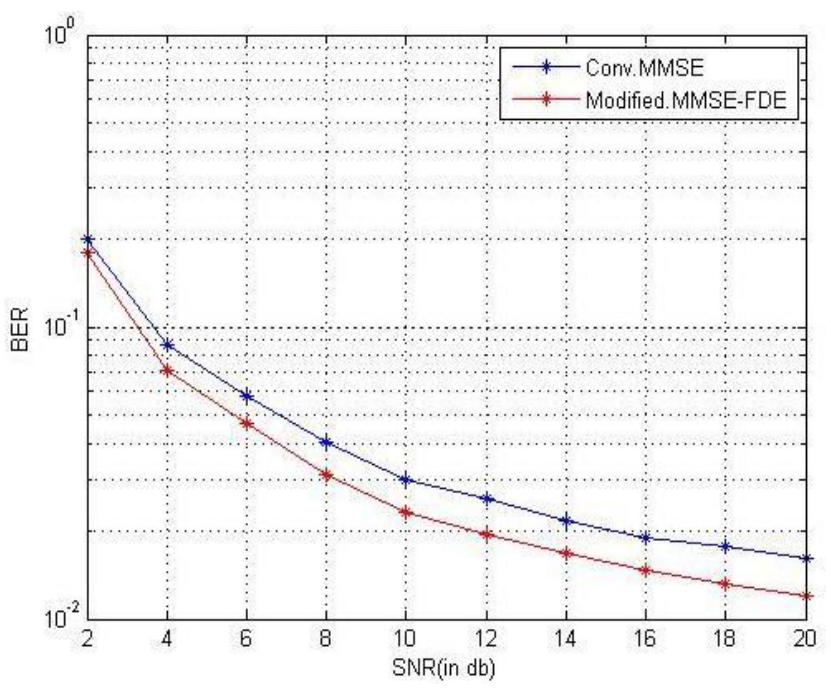

Fig .5 BER Performance of the conventional MMSE equalizer \& Modified MMSE-FDE technique for 64Subcarriers.

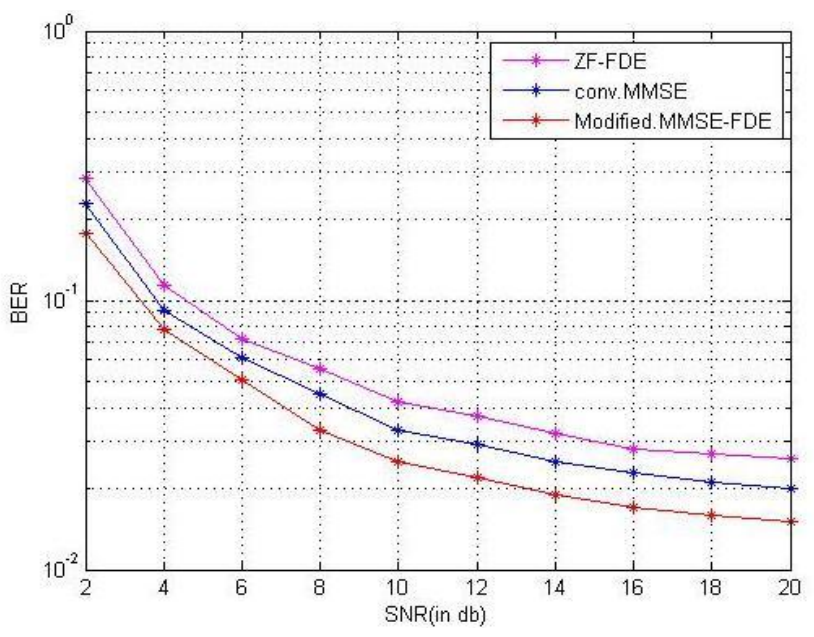

Fig 6: BER performance of the ZF-FDE, conventional MMSE equalization and the Modified MMSE-FDE equalizer for 32-Subcarriers.

\section{CONCLUSION}

In this paper the ZF-FDE, Conventional MMSE equalization and Modified MMSE-FDE are analyzed for MC-CDMA system. From the simulation, it is shown that the Modified MMSE-FDE achieves improved performance over ZF-FDE and conventional MMSE equalization. Modified MMSEFDE takes into account the remaining MUI term for designing the FDE coefficients and thereby reduces the interference among the users caused due to CFO, which occurs due to Doppler shift or asynchrony among transmit and receive oscillator. Thus it evidently restores the orthogonality among the subcarriers. The performance of Modified MMSE-FDE analyzed with different subcarriers, even with which the Modified MMSE-FDE provides better performance. So the Modified MMSE-FDE is more suitable equalization technique for MC-CDMA uplink system.

\section{REFERENCES}

[1] Khaled Fazel and Stefan Kaiser,"Multi-Carrier and Spread Spectrum Systems: From OFDM and MCCDMA to LTE and WiMAX" 2/e, John Wiley and Sons Ltd, 2008

[2] Younsun Kim, Sooyong Choi, Chulwoo You, and Daesik Hong , "BER Computation of an MC-CDMA system with carrier frequency offset" proceeding ofIEEE International conference on Acoustics, Speech and Signal Processing (ICCASSP-99), vol.5, pp.25552558,1999 .

[3] Jiho Jang and Kwang Bok (Ed) Lee, "Effects of frequency offset on MC-CDMA system performance" IEEE Communication Letter, Vol.3, No.7, pp.196-198, July 1999Tavel, P. 2007 Modeling and Simulation Design. AK Peters Ltd.

[4] Youssef Nasser, Mathieu des Noes, Laurent Ros and Genevieve Jourdain, "Sensitivity of Multicarrier TwoDimensional Spreading Schemes to Synchronization Errors", EURASIP Journal on Wireless Communication and Networking, Vol.2008, article id 561869.

[5] Ying Li and Xiang Gui, "Effect of Synchronization Errors on the Performance of Multicarrier CDMA Systems", Journal of Communication and Networks, Vol.8, No.1, pp.38-48, March 2006.

[6] Luciano Tomba and Witold A. Krzymien, "Effect of Carrier Phase Noise and Frequency Offset on the Performance of Multicarrier CDMA Systems" Proceeding of IEEE International Conference on Conference Record, Convergence Technologies for Tomorrow's Application (ICC'96), pp. 1513-1517, 1996.

[7] PH Reddy and VU Reddy, "BER Degradation of MCCDMA at High SNR with MMSE Equalization and Residual Frequency Offset", EURASIP Journal on Wireless Communications and Networking, Vol.2009, article ID 293264.

[8] Meng-Lin Kut and Huan-lung Yeh, "An Iterative ICI Equalization and Cancellation Scheme for LTE SCFDMA Systems in Multipath Channels"Proceeding of 12th International Conference on ITS Telecommunications, pp.610-615, 2012 